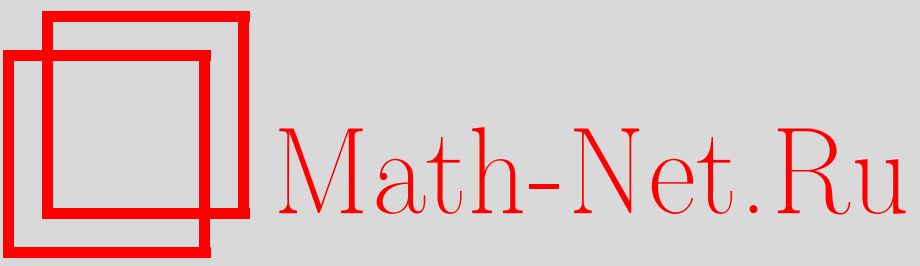

Е. Зеррик, А. Аит Аади, Р. Лариши, Задача регионального оптимального управления вибрирующей пластиной, Итоги науки и техн. Сер. Соврем. мат. и ее прил. Темат. обз., 2020, том 178, 20-30

DOI: https://doi.org/10.36535/0233-6723-2020-178-20-30

Использование Общероссийского математического портала Math-Net.Ru подразумевает, что вы прочитали и согласны с пользовательским соглашением

http://www.mathnet.ru/rus/agreement

Параметры загрузки:

IP : 18.208 .226 .222

26 апреля 2023 г., 09:31:23 


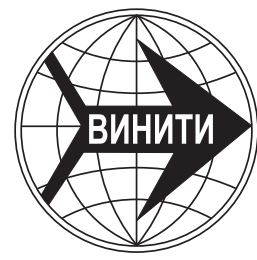

ИТОГИ НАУКИ И ТЕХНИКИ.

Современная математика и ее приложения.

Тематические обзоры.

Том 178 (2020). C. $20-30$

DOI: $10.36535 / 0233-6723-2020-178-20-30$

УДК 517.977.56

\title{
ЗАДАЧА РЕГИОНАЛЬНОГО ОПТИМАЛЬНОГО УПРАВЛЕНИЯ ВИБРИРУЮЩЕЙ ПЛАСТИНОЙ
}

\author{
(c) 2020 г. $\quad$ Е. ЗЕРРИК, А. АИТ ААДИ, Р. ЛАРИШИ
}

\begin{abstract}
АннотАция. Целью работы является изучение задачи регионального оптимального управления вибрирующей пластиной, колеблющейся в пространственной области $\Omega$. Задача состоит в поиске ограниченного управления, под действием которого система, ограниченная на подобласть $\omega \subset \Omega$, переходит за конечное время из заданного начального состояния в желаемое конечное состояние. Доказано существование оптимального управления и установлены его свойства. Приведено условие, обеспечивающее единственность оптимального управления, и предложен численный алгоритм решения.
\end{abstract}

Ключевые слова: распределенная билинейная система, уравнение колебаний пластины, региональная управляемость, оптимальное управление.

\section{REGIONAL OPTIMAL CONTROL PROBLEM FOR A VIBRATING PLATE}

\author{
(C) 2020 E. ZERRIK, A. AIT AADI, R. LARHRISSI
}

\begin{abstract}
In this paper, we examine the problem on the regional optimal control of a vibrating plate in a spatial domain $\Omega$. We obtain a bounded control that drives such a system from an initial state to a desired state in a finite time, only on a subdomain $\omega$ of $\Omega$. We prove that a regional optimal control exists characterize this control. Also we propose a condition that ensures the uniqueness of an optimal control and develop an algorithm for numerical simulations.
\end{abstract}

Keywords and phrases: distributed bilinear system, plate equation, regional controllability, optimal control.

AMS Subject Classification: 93C20, 93D15

1. Введение. Билинейные системы представляют собой специальный класс нелинейных систем, в которых нелинейные члены строятся путем умножения управляющего вектора на вектор состояния. Важность таких систем обусловлена тем, что многие процессы не только в области инженерии, но и в биологии, социоэкономике и химии могут быть смоделированы билинейными системами. Основной результат по управляемости билинейных систем, полученный в [2], показывает, что при некоторых условиях существует мягкое решение (mild solution), связанное с управлением, а множество достижимости из исходного состояния имеет плотное дополнение в пространстве состояний. Это делает сложным нахождение точного решения задачи управления. Большинство результатов было получено для конкретных билинейных систем (см. $[2,7,9])$. Точная управляемость вибрирующих пластин, моделируемых линейными системами, была установлена Ж. Л. Лионсом (см. [10]), использовавшим метод единственности Гильберта (HUM method); Э. Зуазуа (см. [14]) рассмотрел точную управляемость за сколь угодно малое время.

Работа выполнена при поддержке гранта Академии наук и технологий им. Хасана II.. 
Оптимальное управление билинейными системами изучалось во многих работах. М. Брэдли и С. Ленхарт (см. [3-5]) установили существование оптимального управления и однозначно его охарактеризовали через решение оптимальной системы, С. Ленхарт (см. [8]) показал существование и дал характеристику оптимального управления в задаче конвективно-диффузионного переноса жидкости, А. Адду и А. Бенбрик (см. [1]) изучили существование и единственность оптимального управления для класса билинейных систем.

Концепция региональной управляемости для распределенных линейных систем, эволюционирующих в пространственной области $\Omega$, была разработана А. Эль Джаем и Э. Зерриком и относится к изучению классического понятия управляемости только на подобласти $\omega$ в $\Omega$. Основные причины для рассмотрения этого понятия заключаются в том, что оно близко к реальным приложениям, например, к моделированию туннельной печи, когда необходимо поддерживать заданную температуру только в ее части. Кроме того, возможно регионально управлять системой, действуя управлением только на часть $\Omega \backslash \omega$, и существуют системы, управляемые на некоторой подобласти $\omega \subset \Omega$, но неуправляемые на всей области $\Omega$ (см. [6]). Помимо этого, региональное управление системой дешевле глобального управления (см. [6]).

Региональное оптимальное управление распределенными билинейными системами с неограниченным и ограниченным управлением, включающее в себя минимизацию нормы управления и ошибки конечного состояния, было рассмотрено в [12]. В [13]) при помощи уравнения Риккати была рассмотрена задача с неограниченным управлением. В данной работе рассматривается задача регионального оптимального управления вибрирующей пластиной с ограниченным управлением.

Более точно, пусть $\Omega$ - открытая ограниченная область $\mathbb{R}^{2}$ с регулярной границей $\partial \Omega$. Пусть $T>0$; введем обозначение $Q=\Omega \times] 0, T[, \Sigma=\partial \Omega \times[0, T]$ и рассмотрим уравнение колебаний пластины

$$
\begin{cases}\frac{\partial^{2} z(x, t)}{\partial t^{2}}+\Delta^{2} z(x, t)=u(x, t) z(x, t) & \text { в } Q, \\ z(x, 0)=z_{0}(x), \quad \frac{\partial z(x, 0)}{\partial t}=z_{1}(x) & \text { в } \Omega, \\ z(x, t)=\frac{\partial z(x, t)}{\partial \nu}=0 & \text { на } \Sigma,\end{cases}
$$

где $\nu$ - единичный вектор нормали к границе $\partial \Omega, A z=\Delta^{2} z$-неограниченный оператор с областью определения $\mathcal{D}(A)=H^{4}(\Omega) \cap H_{0}^{2}(\Omega)$ и $U_{M}=\left\{u \in L^{\infty}(Q) \mid-M \leqslant u(x, t) \leqslant M\right\}$ - множество управлений, ограниченное положительной константой $M$.

Известно, что при $\tilde{z}(0)=\left(z_{0}, z_{1}\right) \in H_{0}^{2}(\Omega) \times L^{2}(\Omega)$ и $u \in U_{M}$ система (1) имеет единственное слабое решение $\tilde{z}(u)=\left(z, z_{t}\right)$, где $\left(z, z_{t}\right) \in \mathcal{C}\left([0, T], H_{0}^{2}(\Omega) \times L^{2}(\Omega)\right)$ (см. [3]). Рассмотрим непустое подмножество $\omega \subset \Omega$ с положительной мерой Лебега и желаемое конечное состояние $z_{d}$ в $L^{2}(\omega)$.

Исследуется следующая задача регионального оптимального управления вибрирующей пластиной:

$$
\min _{u \in U_{M}} J(u)
$$

где

$$
J(u)=\frac{1}{2} \int_{\omega} \int_{0}^{T}\left(\chi_{\omega} z(x, t)-z_{d}(x)\right)^{2} d t d x+\frac{\beta}{2} \int_{Q} u^{2}(x, t) d x d t,
$$

где $\beta$ - положительная константа, $\chi_{\omega}: L^{2}(\Omega) \rightarrow L^{2}(\omega)$ - оператор сужения на $\omega, \chi_{\omega}^{*}$ - сопряженный оператор к $\chi_{\omega}$, определяемый формулой

$$
\chi_{\omega}^{*} z(x)= \begin{cases}z(x) & \text { при } x \in \omega, \\ 0 & \text { при } x \in \Omega \backslash \omega .\end{cases}
$$

Цель данной работы - охарактеризовать оптимальное управление $u^{*} \in U_{M}$ в задаче (2). Структура работы такова. В разделе 2 установлено существование оптимального управления с помощью минимизирующей последовательности и дана его характеристика, основанная на решении оптимальной системы, состоящей из системы (1) и сопряженной системы. В разделе 3 изучена 
единственность оптимального управления. В разделе 4 предложен численный метод и алгоритм, приведены результаты численного моделирования.

\section{2. Существование и характеристика оптимального управления.}

Теорема 1. Существует оптимальное управление $u^{*} \in U_{M}$, решающее задачу (2).

Доказательство. Пусть $\left(u_{n}\right) \in U_{M}$ - такая минимизирующая последовательность, что

$$
\lim _{n \rightarrow \infty} J\left(u_{n}\right)=\inf _{u \in U_{M}} J(u) .
$$

Соответствующее решение системы (1) обозначим через $\tilde{z}_{n}=\tilde{z}\left(u_{n}\right)$. В силу [3, лемма 2.2] имеем

$$
\left\|\tilde{z}_{n}\right\|_{\mathcal{C}\left([0, T] ; H_{0}^{2}(\Omega) \times L^{2}(\Omega)\right)} \leqslant C_{1} e^{C_{2} M T}
$$

где $C_{1}=\left\|\tilde{z_{0}}\right\|_{H_{0}^{2}(\Omega) \times L^{2}(\Omega)}$; поскольку $U_{M}$ - замкнутое выпуклое множество в сильной топологии в $L^{2}(Q)$, то оно замкнуто и в слабой топологии в $L^{2}(Q)$. Тогда выполнены следующие свойства сходимости:

$$
\begin{array}{ll}
z_{n} \rightarrow z^{*} & \text { слабо в } L^{2}\left([0, T], H_{0}^{2}(\Omega)\right), \\
z_{n} \rightarrow z^{*} & \text { сильно в } L^{2}(Q), \\
\dot{z}_{n} \rightarrow \dot{z}^{*} & \text { слабо в } L^{2}(Q), \\
u_{n} \rightarrow u^{*} & \text { слабо в } L^{2}(Q) .
\end{array}
$$

Теперь в системе (1) можно перейти к пределу при $n \rightarrow \infty$ и получить, что $\tilde{z}=\tilde{z}(u)=\left(z^{*}, z_{t}^{*}\right)$ решает систему (1) с управлением $u^{*}$. Так как функционал $J$ полунепрерывен снизу относительно слабой сходимости (лемма Фату, см. [11]), получаем

$$
J\left(u^{*}\right) \leqslant \lim _{n \rightarrow \infty} \inf J\left(u_{n}\right)=\inf _{u \in U_{M}} J(u)
$$

откуда заключаем, что $u^{*}$ является решением задачи (2).

Чтобы получить характеристику оптимального управления, мы должны построить оптимальную систему, дифференцируя функционал стоимости $J(u)$ по управлению $u$. Исследуем дифференцируемость $u \rightarrow \tilde{z}(u)$ относительно $u$.

Лемма 1. Отображение $u \in U_{M} \rightarrow \tilde{z}(u) \in H_{0}^{2}(\Omega) \times L^{2}(\Omega)$ является дифберенцируемым 8 следуюшем смысле:

$$
\frac{\tilde{z}(u+\varepsilon h)-\tilde{z}(u)}{\varepsilon} \rightarrow \tilde{\psi} \quad \text { слабо в } H_{0}^{2}(\Omega) \times L^{2}(\Omega) \text { при } \varepsilon \rightarrow 0 \text { для любых } u, u+\varepsilon h \in U_{M} .
$$

Кроме того, $\tilde{\psi}=\left(\psi, \psi_{t}\right)$ является слабым решением системы

$$
\begin{cases}\frac{\partial^{2} \psi(x, t)}{\partial t^{2}}+\Delta^{2} \psi(x, t)=u(x, t) \psi(x, t)+h(x, t) z(x, t) & \text { в } Q, \\ \psi(x, 0)=\frac{\partial \psi(x, 0)}{\partial t}=0 & \text { в } \Omega, \\ \psi(x, t)=\frac{\partial \psi(x, t)}{\partial \nu}=0 & \text { на } \Sigma,\end{cases}
$$

әде $\tilde{z}=\tilde{z}(u)=\left(z, z_{t}\right)$. 
Доказательство. Введем обозначения $\tilde{z}^{\varepsilon}=\tilde{z}(u+\varepsilon h)=\left(z^{\varepsilon}, z_{t}^{\varepsilon}\right)$ и $\tilde{z}=\tilde{z}(u)$. Тогда $\left(\tilde{z}^{\varepsilon}-\tilde{z}\right) / \varepsilon$ является слабым решением системы

$$
\begin{cases}\frac{\partial^{2}}{\partial t^{2}}\left(\frac{z^{\varepsilon}-z}{\varepsilon}\right)+\Delta^{2}\left(\frac{z^{\varepsilon}-z}{\varepsilon}\right)=u\left(\frac{z^{\varepsilon}-z}{\varepsilon}\right)+h z^{\varepsilon} & \text { в } Q, \\ \left(\frac{z^{\varepsilon}-z}{\varepsilon}\right)(x, 0)=\frac{\partial}{\partial t}\left(\frac{z^{\varepsilon}-z}{\varepsilon}\right)(x, 0)=0 & \text { в } \Omega, \\ \left(\frac{z^{\varepsilon}-z}{\varepsilon}\right)=\frac{\partial}{\partial \nu}\left(\frac{z^{\varepsilon}-z}{\varepsilon}\right)=0 & \text { на } \Sigma .\end{cases}
$$

Используя [3, лемма 2.2], получаем

$$
\frac{1}{\varepsilon}\left\|\tilde{z}^{\varepsilon}-\tilde{z}\right\|_{\mathcal{C}\left([0, T] ; H_{0}^{2}(\Omega) \times L^{2}(\Omega)\right)} \leqslant\left\|h z^{\varepsilon}\right\|_{L^{2}(Q)} e^{C M T} \leqslant C_{3},
$$

где $C_{3}$ не зависит от $\varepsilon$, поскольку оценка на $\left\|\tilde{z}^{\varepsilon}\right\|_{L^{2}(Q)}$ не зависит от $\varepsilon$. Таким образом, имеем

$$
\frac{\tilde{z}^{\varepsilon}-\tilde{z}}{\varepsilon} \rightarrow \tilde{\psi} \quad \text { слабо в } H_{0}^{2}(\Omega) \times L^{2}(\Omega) \text { при } \varepsilon \rightarrow 0 .
$$

Заключаем, что $\psi$ является слабым решением системы (4).

Теперь есть все необходимое, чтобы вывести оптимальную систему (путем дифференцирования $J(u)$ по $u)$ и тем самым охарактеризовать оптимальное управление.

Теорема 2. Для заданного оптимального управления $u^{*} \in U_{M}$ и соответствующего ему решения $\tilde{z}=\tilde{z}\left(u^{*}\right)=\left(z^{*}, z_{t}^{*}\right)$ системы (1) сопряжсенная система

$$
\begin{cases}\frac{\partial^{2} p(x, t)}{\partial t^{2}}+\Delta^{2} p(x, t)=u^{*}(x, t) p(x, t)+z^{*}(x, t)-\chi_{\omega}^{*} z_{d}(x) & \text { в } Q, \\ p(x, T)=\frac{\partial p(x, T)}{\partial t}=0 & \text { в } \Omega, \\ p(x, t)=\frac{\partial p(x, t)}{\partial \nu}=0 & \text { на } \Sigma\end{cases}
$$

имеет единственное слабое решение $\tilde{p}=\left(p, p_{t}\right) \in \mathcal{C}\left([0, T], H_{0}^{2}(\Omega) \times L^{2}(\Omega)\right)$. Кроме того,

$$
u^{*}(x, t)=\max \left(M, \min \left(-\frac{1}{\beta} z^{*}(x, t) \chi_{\omega}^{*} \chi_{\omega} p(x, t), M\right)\right) .
$$

Доказательство. Пусть $u^{*} \in U_{M}$ - оптимальное управление, а $\tilde{z}=\tilde{z}\left(u^{*}\right)$ - соответствующее оптимальное решение. Пусть $u^{*}+\varepsilon h \in U_{M}$ для $\varepsilon>0$ и $\tilde{z}^{\varepsilon}=\tilde{z}\left(u^{*}+\varepsilon h\right)$ - соответствующее слабое решение системы (1). Вычислим производную функционала $J$ относительно $u^{*}$ по направлению $h$. Так как $J$ должен достичь минимума при $u^{*}$, имеем

$$
\begin{aligned}
& 0 \leqslant \lim _{\varepsilon \rightarrow 0^{+}} \frac{J\left(u^{*}+\varepsilon h\right)-J\left(u^{*}\right)}{\varepsilon}= \\
&=\lim _{\varepsilon \rightarrow 0^{+}} \frac{1}{2} \int_{\omega} \int_{0}^{T} \frac{\left(\chi_{\omega} z^{\varepsilon}-z_{d}\right)^{2}-\left(\chi_{\omega} z^{*}-z_{d}\right)^{2}}{\varepsilon} d x d t+\lim _{\varepsilon \rightarrow 0^{+}} \frac{\beta}{2} \int_{Q} \frac{\left(u^{*}+\varepsilon h\right)^{2}-u^{* 2}}{\varepsilon} d x d t= \\
&=\lim _{\varepsilon \rightarrow 0^{+}} \frac{1}{2} \int_{\omega} \int_{0}^{T} \chi_{\omega} \frac{\left(z^{\varepsilon}-z^{*}\right)}{\varepsilon}\left(\chi_{\omega} z^{\varepsilon}+\chi_{\omega} z^{*}-2 z_{d}\right) d x d t+\lim _{\varepsilon \rightarrow 0^{+}} \frac{\beta}{2} \int_{Q}\left(2 h u^{*}+\varepsilon h^{2}\right) d x d t .
\end{aligned}
$$

Тогда

$$
\lim _{\varepsilon \rightarrow 0^{+}} \frac{J\left(u^{*}+\varepsilon h\right)-J\left(u^{*}\right)}{\varepsilon}=\int_{Q} \chi_{\omega}^{*} \chi_{\omega} \psi\left(z^{*}-\chi_{\omega}^{*} z_{d}\right) d x d t+\beta \int_{Q} h u^{*} d x d t,
$$

где $\psi$-решение системы (4). 
Используя сопряженную систему (5) и $\psi$ как решение системы (4), получаем

$$
\begin{aligned}
0 \leqslant \int_{\Omega} \chi_{\omega}^{*} \chi_{\omega} \int_{0}^{T} \psi\left(\frac{\partial^{2} p}{d t^{2}}+\Delta^{2} p-u p\right) d t d x+\beta \int_{Q} h u^{*} d x d t= \\
=\int_{\Omega} \chi_{\omega}^{*} \chi_{\omega} \int_{0}^{T}\left(\frac{\partial^{2} \psi}{d t^{2}}+\Delta^{2} \psi-u \psi\right) p d t d x+\beta \int_{Q} h u^{*} d x d t= \\
=\int_{\Omega} \chi_{\omega}^{*} \chi_{\omega} \int_{0}^{T} h z^{*} p d t d x+\beta \int_{Q} h u^{*} d t d x=\int_{Q} h\left(\beta u^{*}+\chi_{\omega}^{*} \chi_{\omega} z^{*} p\right) d x d t= \\
=\int_{Q} h\left(\beta u^{*}+z^{*} \chi_{\omega}^{*} \chi_{\omega} p\right) d x d t
\end{aligned}
$$

Таким образом, используя произвольную вариацию $h$ и оценки на множество управлений $U_{M}$, получаем

Теорема доказана.

$$
u^{*}(x, t)=\max \left(-M, \min \left(-\frac{1}{\beta} z^{*}(x, t) \chi_{\omega}^{*} \chi_{\omega} p(x, t), M\right)\right) .
$$

Следствие 1. В предположении, что $u^{*}(x, t)=v^{*}(t) \mathbb{1}_{D}(x)$, где $D \subset \Omega-$ область приложения управления, оптимальное управление $v^{*}$ имеет вид

$$
v^{*}(t)=\max \left(-M, \min \left(-\frac{1}{\beta \mu(D)} \int_{\Omega} z^{*}(x, t) \chi_{\omega}^{*} \chi_{\omega} p(x, t) d x, M\right)\right),
$$

где $\mu(D)$ - мера Лебега $D, z^{*}$ является решением системъ

$$
\begin{cases}\frac{\partial^{2} z(x, t)}{\partial t^{2}}+\Delta^{2} z(x, t)=v^{*}(t) \mathbb{1}_{D}(x) z(x, t) & \text { в } Q \\ z(x, 0)=z_{0}(x), \quad \frac{\partial z(x, 0)}{\partial t}=z_{1}(x) & \text { в } \Omega, \\ z(x, t)=\frac{\partial z(x, t)}{\partial \nu}=0 & \text { на } \Sigma,\end{cases}
$$

а р является решением сопряженной системы

$$
\left\{\begin{array}{lc}
\frac{\partial^{2} p(x, t)}{\partial t^{2}}+\Delta^{2} p(x, t)=v^{*}(t) \mathbb{1}_{D}(x) p(x, t)+z^{*}(x, t)-\chi_{\omega}^{*} z_{d}(x) & \text { в } Q \\
p(x, T)=\frac{\partial p(x, T)}{\partial t}=0 & \text { в } \Omega \\
p(x, t)=\frac{\partial p(x, t)}{\partial \nu}=0 & \text { на } \Sigma .
\end{array}\right.
$$

Замечание 1. Ясно, что оптимальное управление (7) зависит от области $\omega$ и области приложения управления $D$.

3. Единственность оптимального управления. В этом разделе приведены достаточные условия единственности оптимального управления в задаче (2).

Теорема 3. При достаточно малом Т оптимальное управление (6) в задаче (2) единственно. Доказательство. Предположим, что существуют два слабых решения, соответствующих оптимальным управлениям $u$ и $\bar{u}$ :

$$
\tilde{z}=\left(z, z_{t}\right), \quad \tilde{p}=\left(p, p_{t}\right), \quad \hat{z}=\left(\bar{z}, \bar{z}_{t}\right), \quad \hat{p}=\left(\bar{p}, \bar{p}_{t}\right),
$$


причем $z, p, \bar{z}$ и $\bar{p}$ ограничены в $Q$. Обозначим через $\lambda$ положительную постоянную, которая будет выбрана позже, и выполним замену переменных

$$
z=e^{\lambda t} w, \quad p=e^{-\lambda t} q, \quad \bar{z}=e^{\lambda t} \bar{w}, \quad \bar{p}=e^{-\lambda t} \bar{q} .
$$

Тогда $w$ и $q$ удовлетворяют в слабом смысле следующей системе уравнений:

$$
\left\{\begin{array}{lr}
w_{t t}+2 \lambda w_{t}+\lambda^{2} w+\Delta^{2} w=\max \left(-M, \min \left(-\frac{1}{\beta} w \chi_{\omega}^{*} \chi_{\omega} q, M\right)\right) w & \text { в } Q, \\
q_{t t}-2 \lambda q_{t}+\lambda^{2} q+\Delta^{2} q=\max \left(-M, \min \left(-\frac{1}{\beta} w \chi_{\omega}^{*} \chi_{\omega} q, M\right)\right) q+e^{2 \lambda t} w-e^{\lambda t} \chi_{\omega}^{*} z_{d} & \text { в } Q, \\
w=q=\frac{\partial w}{\partial \nu}=\frac{\partial q}{\partial \nu}=0 & \text { на } \Sigma, \\
w(x, 0)=z_{0}(x), \quad \frac{\partial w(x, 0)}{\partial t}=z_{1}(x) & \text { в } \Omega, \\
q(x, T)=\frac{\partial q(x, T)}{\partial t}=0 & \text { в } \Omega .
\end{array}\right.
$$

Аналогично, $\bar{w}$ и $\bar{q}$ удовлетворяют системе

$$
\left\{\begin{array}{lc}
\bar{w}_{t t}+2 \lambda \bar{w}_{t}+\lambda^{2} \bar{w}+\Delta^{2} \bar{w}=\max \left(-M, \min \left(-\frac{1}{\beta} \bar{w} \chi_{\omega}^{*} \chi_{\omega} \bar{q}, M\right)\right) \bar{w} & \text { в } Q, \\
\bar{q}_{t t}-2 \lambda \bar{q}_{t}+\lambda^{2} \bar{q}+\Delta^{2} \bar{q}=\max \left(-M, \min \left(-\frac{1}{\beta} \bar{w} \chi_{\omega}^{*} \chi_{\omega} \bar{q}, M\right)\right) \bar{q}+e^{2 \lambda t} \bar{w}-e^{\lambda t} \chi_{\omega}^{*} z_{d} & \text { в } Q, \\
\bar{w}=\bar{q}=\frac{\partial \bar{w}}{\partial \nu}=\frac{\partial \bar{q}}{\partial \nu}=0 & \text { на } \Sigma, \\
\bar{w}(x, 0)=z_{0}(x), \quad \frac{\partial \bar{w}(x, 0)}{\partial t}=z_{1}(x) & \text { в } \Omega, \\
\bar{q}(x, T)=\frac{\partial \bar{q}(x, T)}{\partial t}=0 & \text { в } \Omega
\end{array}\right.
$$

Воспользовавшись (10) и (11), получаем

$$
\begin{aligned}
& 0=(w-\bar{w})_{t t}+2 \lambda(w-\bar{w})_{t}+\lambda^{2}(w-\bar{w})+\Delta^{2}(w-\bar{w})-u w+\bar{u} \bar{w} \\
& 0=-(q-\bar{q})_{t t}+2 \lambda(q-\bar{q})_{t}-\lambda^{2}(q-\bar{q})-\Delta^{2}(q-\bar{q})+u q-\bar{u} \bar{q}+e^{2 \lambda t}(w-\bar{w}) .
\end{aligned}
$$

Умножая уравнение $(12)$ на $(w-\bar{w})_{t}$, уравнение $(13)$ на $(q-\bar{q})_{t}$, а затем интегрируя по $Q$, получаем

$$
\begin{gathered}
0=\int_{Q}\left[(w-\bar{w})_{t t}(w-\bar{w})_{t}+2 \lambda(w-\bar{w})_{t}^{2}+\lambda^{2}(w-\bar{w})(w-\bar{w})_{t}+\right. \\
\left.\quad+\Delta(w-\bar{w}) \Delta(w-\bar{w})_{t}-(u w-\bar{u} \bar{w})(w-\bar{w})_{t}\right] d x d t \\
0=\int_{Q}\left[-(q-\bar{q})_{t t}(q-\bar{q})_{t}+2 \lambda(q-\bar{q})_{t}^{2}-\lambda^{2}(q-\bar{q})(q-\bar{q})_{t}-\right. \\
\left.-\Delta(q-\bar{q}) \Delta(q-\bar{q})_{t}+(u q-\bar{u} \bar{q})(q-\bar{q})_{t}+e^{2 \lambda t}(w-\bar{w})(q-\bar{q})_{t}\right] d x d t
\end{gathered}
$$

где

$$
u=\max \left(-M, \min \left(-\frac{1}{\beta} w \chi_{\omega}^{*} \chi_{\omega} q, M\right)\right), \quad \bar{u}=\max \left(-M, \min \left(-\frac{1}{\beta} \bar{w} \chi_{\omega}^{*} \chi_{\omega} \bar{q}, M\right)\right) .
$$


Комбинируя уравнения (14), (15) и используя начальные и граничные условия, получаем

$$
\begin{gathered}
\frac{1}{2} \int_{\Omega}\left[\left((w-\bar{w})_{t}\right)^{2}(x, T)+\left((q-\bar{q})_{t}\right)^{2}(x, 0)\right] d x+\frac{\lambda^{2}}{2} \int_{\Omega}\left[(w-\bar{w})^{2}(x, T)+(q-\bar{q})^{2}(x, 0)\right] d x+ \\
+\int_{\Omega}\left[|\Delta(w-\bar{w})|^{2}(x, T)+|\Delta(q-\bar{q})|^{2}(x, 0)\right] d x+2 \lambda \int_{Q}\left[\left((q-\bar{q})_{t}\right)^{2}+\left((w-\bar{w})_{t}\right)^{2}\right] d x d t= \\
=\int_{Q}\left[(u w-\bar{u} \bar{w})(w-\bar{w})_{t}-(u q-\bar{u} \bar{q})(q-\bar{q})_{t}-e^{2 \lambda t}(w-\bar{w})(q-\bar{q})_{t}\right] d x d t .
\end{gathered}
$$

В силу соотношений

$$
u w-\bar{u} \bar{w}=u(w-\bar{w})+\bar{w}(u-\bar{u}), \quad u q-\bar{u} \bar{q}=u(q-\bar{q})+\bar{q}(u-\bar{u})
$$

и неравенства

$$
|u-\bar{u}| \leqslant \frac{1}{\beta}\left|\bar{w} \chi_{\omega}^{*} \chi_{\omega} \bar{q}-w \chi_{\omega}^{*} \chi_{\omega} q\right|
$$

из (16) следует

$$
\begin{aligned}
2 \lambda \int_{Q}\left[((q-\bar{q}) t)^{2}+\left((w-\bar{w})_{t}\right)^{2}\right] d x d t \leqslant \int_{Q}\left[((q-\bar{q}) t)^{2}+\left((w-\bar{w})_{t}\right)^{2}\right] d x d t+ \\
\quad+C e^{C(M+\lambda) T} \int_{Q}\left[(w-\bar{w})^{2}+(q-\bar{q})^{2}\right] d x d t
\end{aligned}
$$

где $C$ не зависит от $\lambda$ и $T$, но зависит от $L^{\infty}$ ограничений на $z, \bar{p}$. Поскольку

$$
\begin{gathered}
\int_{Q}(w-\bar{w})^{2} d x d t=\int_{\Omega} \int_{0}^{T}\left(\int_{0}^{t}(w-\bar{w})_{t}(x, s) d s\right)^{2} d x d t \leqslant \\
\quad \leqslant \int_{\Omega} \int_{0}^{T} t\left(\int_{0}^{t}\left((w-\bar{w})_{t}\right)^{2}(x, s) d s\right) d x d t \leqslant \frac{T^{2}}{2} \int_{Q}\left((w-\bar{w})_{t}\right)^{2} d x d t,
\end{gathered}
$$

и, аналогично,

$$
\int_{Q}(q-\bar{q})^{2} d x d t \leqslant \frac{T^{2}}{2} \int_{Q}\left((q-\bar{q})_{t}\right)^{2} d x d t
$$

оценка второго слагаемого в (18) влечет

$$
(2 \lambda-1) \int_{Q}\left[\left((q-\bar{q})_{t}\right)^{2}+\left((w-\bar{w})_{t}\right)^{2}\right] d x d t \leqslant T^{2} C e^{C(M+\lambda) T} \int_{Q}\left[\left((q-\bar{q})_{t}\right)^{2}+\left((w-\bar{w})_{t}\right)^{2}\right] d x d t .
$$

Теперь выберем такое $\lambda$, что $2 \lambda-1>0$, и достаточно малое $T$, удовлетворяющее условию

$$
2 \lambda-1>T^{2} C e^{C(M+\lambda) T},
$$

так что

$$
(q-\bar{q})_{t}=(w-\bar{w})_{t}=0 \quad \text { в } Q .
$$

В силу согласования $q, \bar{q}$ и $w, \bar{w}$ на верхней и нижней границах цилиндра $Q$ соответственно, т.е. соотношений $q=\bar{q}, w=\bar{w}$, заключаем, что $u=\bar{u}$. 


\section{4. Алгоритм и численное моделирование.}

4.1. Алгоритм. Мы показали, что решение задачи (2) дается формулой

$$
u^{*}(x, t)=\max \left(-M, \min \left(-\frac{1}{\beta} z^{*}(x, t) \chi_{\omega}^{*} \chi_{\omega} p(x, t), M\right)\right),
$$

где $z^{*}$-решение системы (1), соответствующее управлению $u^{*}$, а $p$-решение системы (5). Оптимальное управление в задаче (2) может быть вычислено по следующей формуле:

$$
u_{n+1}^{*}(x, t)=\max \left(-M, \min \left(-\frac{1}{\beta} z_{n}^{*}(x, t) \chi_{\omega}^{*} \chi_{\omega} p_{n}(x, t), M\right)\right) u_{0}^{*}=0,
$$

где $z_{n}^{*}$-решение системы (1), соответствующее управлению $u_{n}^{*}$, а $p_{n}$-решение сопряженной системы (5). Это позволяет сформулировать следующий алгоритм.

\section{Алгоритм 1.}

Шаг 1: Исходные данные.

(i) Исходное состояние $z_{0}, z_{1}$ и $u_{0}$.

(ii) Желаемое состояние $z_{d}$.

(iii) Пороговая точность $\epsilon$, подобласть $\omega$ и время $T$.

Шаг 2: Повторять, пока выполняется неравенство $\left\|u_{n+1}-u_{n}\right\| \leqslant \epsilon$.

(i) Найти $z_{n}^{*}$, решая систему (1) методом конечных разностей.

(ii) Найти $p_{n}$, решая систему (5) методом конечных разностей.

(iii) Вычислить $u_{n+1}$ по формуле (19).

Шаг 3: Найденное управление $u_{n}^{*}$ оптимально.

4.2. Численное моделирование. Положим $\Omega=] 0,1[\times] 0,1[$. Рассмотрим уравнение колебаний пластины

$$
\begin{cases}\frac{\partial^{2} z\left(x_{1}, x_{2}, t\right)}{\partial t^{2}}+\Delta^{2} z\left(x_{1}, x_{2}, t\right)=v(t) \mathbb{1}_{D}\left(x_{1}, x_{2}\right) z\left(x_{1}, x_{2}, t\right) & \text { в } \Omega \times] 0, T[, \\ z\left(x_{1}, x_{2}, 0\right)=z_{0}\left(x_{1}, x_{2}\right), \quad \frac{\partial z\left(x_{1}, x_{2}, 0\right)}{\partial t}=z_{1}\left(x_{1}, x_{2}\right) & \text { в } \Omega, \\ z\left(x_{1}, x_{2}, t\right)=\frac{\partial z\left(x_{1}, x_{2}, t\right)}{\partial \nu}=0 & \text { на } \Sigma,\end{cases}
$$

и задачу (2) с множеством управлений $U_{M}=\left\{v \in L^{2}(] 0, T[) ;-M \leqslant v(t) \leqslant M\right\}$. Оптимальное управление в задаче (2) задается следующей формулой:

$$
\left\{\begin{array}{l}
v_{n+1}^{*}(t)=\max \left(-M, \min \left(-\frac{1}{\beta \mu(D)} \int_{\Omega} z_{n}^{*}\left(x_{1}, x_{2}, t\right) \chi_{\omega}^{*} \chi_{\omega} p_{n}\left(x_{1}, x_{2}, t\right) d x_{1} d x_{2}, M\right)\right), \\
v_{0}^{*}=0,
\end{array}\right.
$$

где $z_{n}^{*}$-решение системы (20), соответствующее управлению $v_{n}^{*}$, и $p_{n}$-решение сопряженной системы

$$
\left\{\begin{array}{rr}
\frac{\partial^{2} p_{n}\left(x_{1}, x_{2}, t\right)}{\partial t^{2}}+\Delta^{2} p_{n}\left(x_{1}, x_{2}, t\right)= & \text { в } Q, \\
=v^{*}(t) \mathbb{1}_{D}\left(x_{1}, x_{2}\right) p_{n}\left(x_{1}, x_{2}, t\right)+z_{n}^{*}\left(x_{1}, x_{2}, t\right)-\chi_{\omega}^{*} z_{d}\left(x_{1}, x_{2}\right) & \text { в } \Omega, \\
p_{n}\left(x_{1}, x_{2}, T\right)=\frac{\partial p_{n}\left(x_{1}, x_{2}, T\right)}{\partial t}=0 & \text { на } \Sigma . \\
p_{n}\left(x_{1}, x_{2}, t\right)=\frac{\partial p_{n}\left(x_{1}, x_{2}, t\right)}{\partial \nu}=0 & \text {. }
\end{array}\right.
$$

Положим $T=1, M=1, \beta=0.1, z_{0}\left(x_{1}, x_{2}\right)=x_{1} x_{2}\left(1-x_{1}\right)\left(1-x_{2}\right), z_{1}\left(x_{1}, x_{2}\right)=x_{1} x_{2}\left(1-x_{1}\right)\left(1-x_{2}\right)$, желаемое конечное состояние $z_{d}\left(x_{1}, x_{2}\right)=0$ на подобласти $\omega \subset \Omega$ и $\left.D=\right] 0,3 ; 0,6[\times] 0,3 ; 0,6[$. Начальное состояние показано на рис. 1. Применяя предложенный выше алгоритм с $\epsilon=10^{-4}$, получаем следующий результат. 


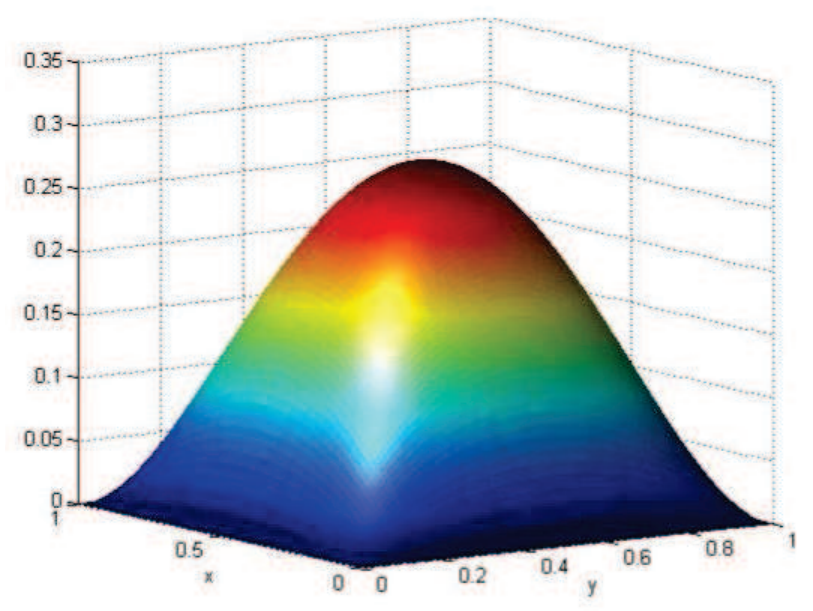

Рис. 1. Начальное состояние на $\Omega$.

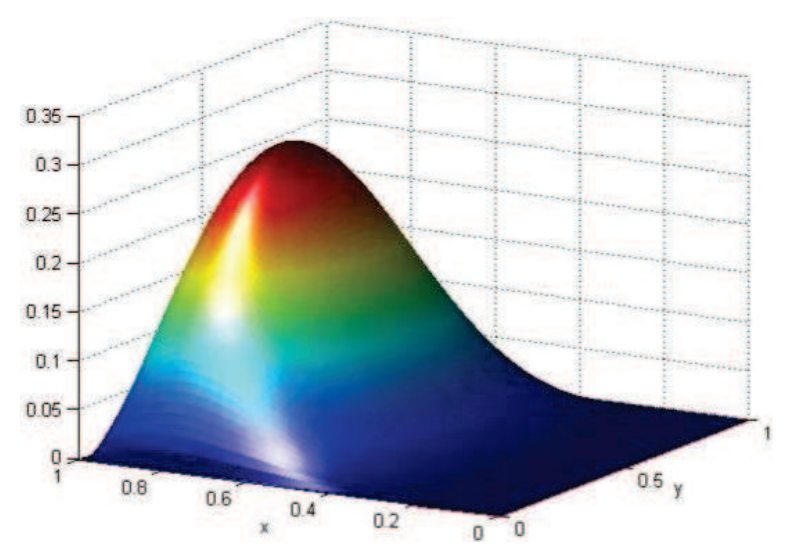

Рис. 2. Конечное состояние на $\Omega$.

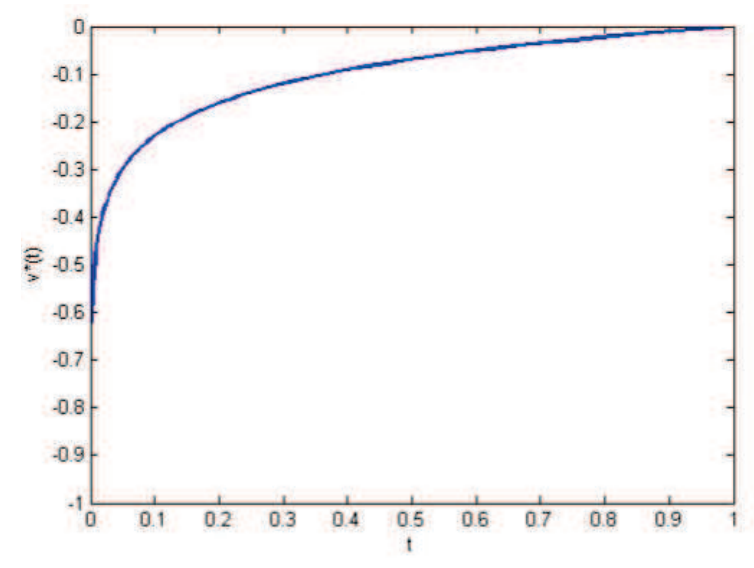

Рис. 3. Оптимальное управление.

А. Результат вычислений для $\omega=] 0 ; 0,2[\times] 0 ; 1[$ изображен на рис. 2 , 3. Желаемое состояние достигнуто с точностью $\left\|\chi_{\omega} z(T)\right\|_{L^{2}(\omega)}^{2}=6,2 \times 10^{-4}$, а значение функционала равно $J\left(v^{*}\right)=$ $2,8 \times 10^{-3}$.

В. Результат вычислений для $\omega=] 0 ; 0,5[\times] 0 ; 1[$ изображен на рис. 4, 5. Желаемое состояние достигнуто с точностью $\left\|\chi_{\omega} z(T)\right\|_{L^{2}(\omega)}^{2}=8,5 \times 10^{-4}$, а значение функционала равно $J\left(v^{*}\right)=$ $5,4 \times 10^{-3}$.

С. Результат вычислений для $\omega=\Omega=] 0 ; 1[\times] 0 ; 1[$ изображен на рис. 6,7 . Желаемое состояние достигнуто с точностью $\left\|\chi_{\omega} z(T)\right\|_{L^{2}(\omega)}^{2}=9,2 \times 10^{-3}$, а значение функционала равно $J\left(v^{*}\right)=$ $3,2 \times 10^{-2}$.

5. Заключение. Рассмотрена задача регионального оптимального управления вибрирующей пластиной при ограниченном множестве управлений. Доказано существование и единственность оптимального управления и дана его характеристика. Предложенный подход позволяет оценить соответствие конечного состояния заданной цели, ограниченной на подмножество в области определения. Полученные результаты успешно протестированы с помощью численных экспериментов. Случай граничной подобласти остается открытым. 


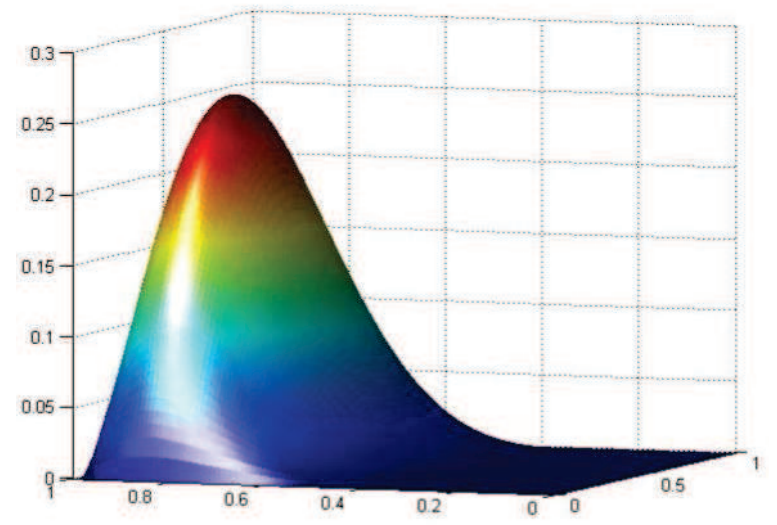

Рис. 4. Конечное состояние на $\Omega$.

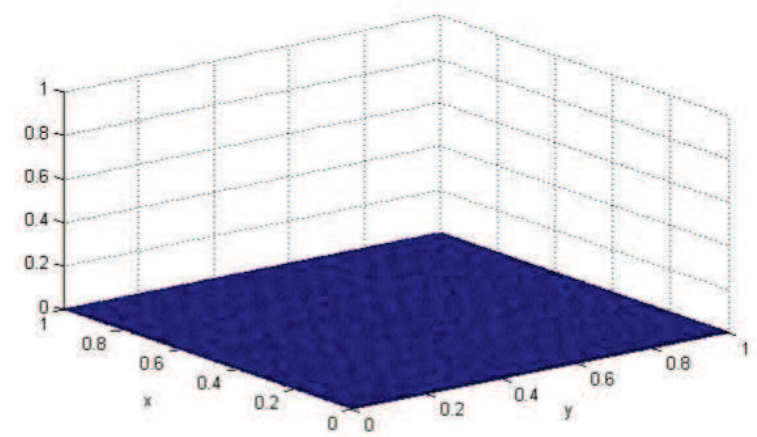

Рис. 6. Конечное состояние на $\Omega$.

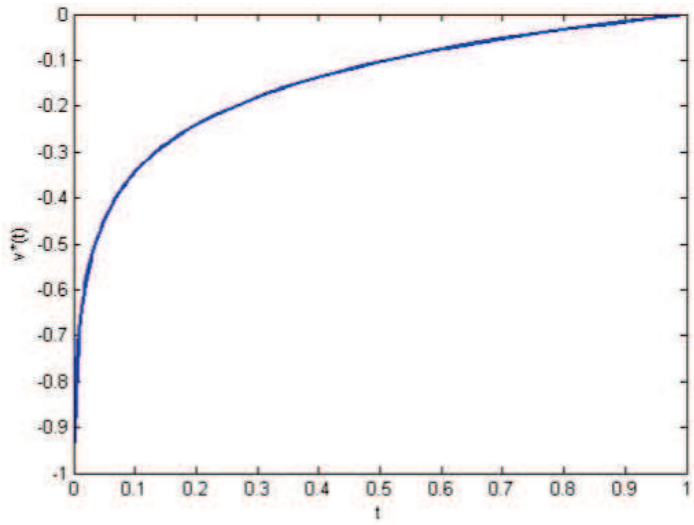

Рис. 5. Оптимальное управление.

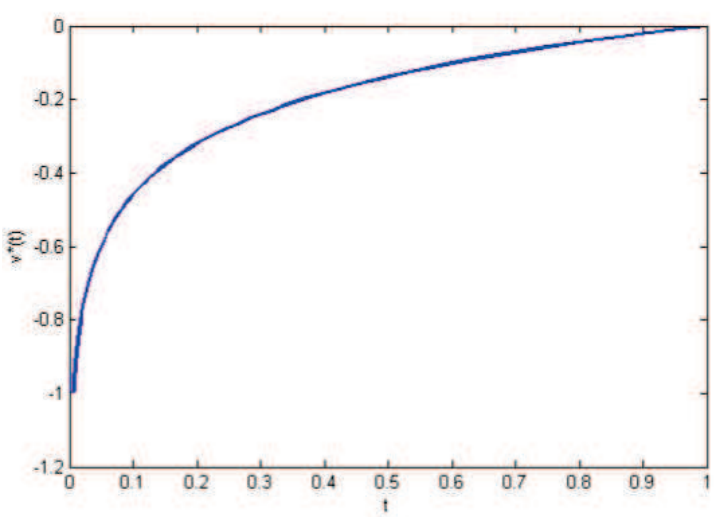

Рис. 7. Оптимальное управление.

\section{СПИСОК ЛИТЕРАТУРЫ}

1. Addou A., Benbrik A. Existence and uniqueness of optimal control for a distributed parameter bilinear systems// J. Dynam. Control Syst. - 2002. - 8. - C. 141-152.

2. Ball J. M., Marsden J. E., and Slemrod M. Controllability for distributed bilinear systems// SIAM J. Control Optim. - 1982. - 20. - C. 575-597.

3. Bradley M. E., Lenhart S. Bilinear optimal control of a Kirchhoff plate// Syst. Control Lett. - 1994. - 22. - C. 27-38.

4. Bradley M. E., Lenhart S., Yong J. Bilinear optimal control of the velocity term in a Kirchhoff plate equation// J. Math. Anal. Appl. - 1999. - 238. - C. 451-467.

5. Bradley M. E., Lenhart S. Bilinear spatial control of the velocity term in a Kirchhoff plate// Electron. J. Differ. Equations. - 2001. - 2001. - 27.

6. El Jai A., Simon M. C., Zerrik E., Prirchard A. J. Regional controllability of distributed parameter systems// Int. J. Control. - 1995. - 62. - C. 1351-1365.

7. Joshi H. R. Optimal control of the convective velocity coefficient in a parabolic problem// Nonlin. Anal. Theory Methods Appl. - 2005. - 63. - C. 1383-1390.

8. Lenhart S. Optimal control of a convective-diffusive fluid problem// J. Math. Models Meth. Appl. Sci. 1995. - 5. - C. 225-237.

9. Liang M. Bilinear optimal control for a wave equation// J. Math. Models Meth. Appl. Sci. - 1999. - 9. C. $45-68$.

10. Lions J. L. Contrôlabilité exacte, perturbations et stabilisation de systèmes distribués. - Paris: Masson, 1988. 
11. Rudin W. Real and Complex analysis. - McGraw-Hill, 1987.

12. Zerrik E., Ould Sidi M. Regional controllability for infinite dimensional distributed bilinear systems// Int. J. Control. - 2011. - 84. - C. 2108-2116.

13. Ztot K., Zerrik E., Bourray H., Regional control problem for distributed bilinear systems// Int. J. Appl. Math. Comput. Sci. - 2011. - 2. - C. 499-508.

14. Zuazua E. Contrôlabilité exacte d'une modèles de plaques en un temps arbitrairement petit// C. R. Acad. Sci. Paris. Ser. I. Math. - 1987. - 304, № 7. - C. 173-176.

Zerrik E.

Университет Мулая Исмаила, Мекнесс, Марокко

E-mail: zerrik3@yahoo.fr

Ait Aadi A.

Университет Мулая Исмаила, Мекнесс, Марокко

E-mail: abderrahman.aitaadi@gmail.com

Larhrissi R.

Университет Мулая Исмаила, Мекнесс, Марокко

E-mail: r.larhrissi@gmail.com 